\title{
Bio Potential Signal Conditioning using MATLAB on Electromyography Signals
}

\author{
Roopa J, Rahul N, Pragna G S, Geetha K S, B S Satyanarayana, Govinda Raju M
}

\begin{abstract}
In today's world of medicine, technology plays a major role in creating solutions. Conventionally expensive equipment has been used for diagnosis involving bio signals. Such treatment is not available to most people. Most existing bio signal conditioning devices are very specific to their application, and others that are diverse require a large number of different sensors and are more expensive and cumbersome to use. There is a need for a signal conditioning circuit that is adaptable, employs digital processing and can be used to measure any of the different bio signals generated by our body with minimal modification. Use of MATLAB can greatly help researchers to analyse bio signals by giving them huge flexibility. MATLAB also has the added advantage of being able to be used on nearly all computing devices. Raw bio signal data has been captured and MATLAB has been used to mimic the various filters required to remove different types of noises that exist in a typical electromyography signal.
\end{abstract}

Keywords-Diagnosis, Signal Conditioning, Digital Processing, MATLAB, Electromyography.

\section{INTRODUCTION}

Electrical activities that are the result of different biological processes in the cells of nervous, muscular or glandular tissue give rise to bio potentials. These are ionic potentials. When adequately stimulated these cells are excitable and are able to conduct an action potential [1]. This flow of the charged ions is sensed using biosensors (electrodes). Bio signals are of paramount importance in modern day medicine. A huge number of diseases require bio signal processing for diagnosis. By trying to achieve precise outputs using MATLAB we can make it easier for students and researchers to effectively perform advanced processing on Bio Signals with ease.

Revised Manuscript Received on July 22, 2019.

Roopa J,

Assistant Professor, ECE Department, RVCE, Bangalore roopaj@rvce.edu.in

Rahul N

U.G. Student, ECE Department, RVCE, Bangalore, India rahulnnarasimha@gmail.com

Pragna G S

U.G. Student, ECE Department, RVCE, Bangalore, India pragnagshastry@gmail.com

Geetha K S

Head of Department, ECE Department, RVCE, Bangalore B S Satyanarayana

Emeritus Prof, Ex-vice Chancellor, BML University, Haryana Govinda Raju M

Assistant Professor, ECE Department, RVCE, Bangalore
EMG signals can be used for diagnosis of diseases associated with muscles including conditions such as paralysis, dysphagia and various other disorders [2]. The motor neurons functionality can be compared to that of a transducer. Through chemical processes it converts neural impulses that it receives from the brain into movement [3]. Being able to effectively process and draw conclusions regarding the health of muscles without the need for having expensive equipment will greatly reduce the cost of physiotherapy and help detect early stages of any sort of muscle ailments. In places where such facilities are not present, raw data can be acquired and analyzed on any computer. The structure of the paper is described in Figure 1.

\section{RELATED WORK}

A. Characteristics of noise associated with EMG Signals

In order for us to remove the noise components from EMG signals we had to make a detailed study on the various types of noises associated with Bio signals

- Inherent Noise: Noise introduced into the signal by the electronic equipment used. They have a wide frequency range and are difficult to eliminate. The only way of reducing such noise is by using careful and deliberate circuit design and instruments of superior quality.

- Electromagnetic Noise: Exposure of the body to electromagnetic radiations from the surrounding environment is inevitable. These radiations superimpose with the signal and result in unwanted electromagnetic noise. They are varied signals and have amplitudes of up to three times the EMG signal [4]. Power Line Interference (PLI) is the dominant source of electromagnetic interference. They exist in the $50 \mathrm{~Hz}$ range of frequency.

- Motion Artifact: Motion artifacts from the electrodes are caused by pressure exerted by the gel layer on the electrode. They have frequency between 1-10 Hz and voltage same as that of the EMG signal. We also need to reduce the motion artifacts caused due to existing differences in potential between layers of the skin. Various techniques are used to remove this type of motion artifact such as skin abrasion before extraction of EMG signals [5].

- ECG Artifacts: The pumping of the heart gives rise to electrocardiographic (ECG) signals. These signals often interfere with EMG signals. The 
effect of these signals can be reduced by common mode rejection. Additional electrodes are used to measure the signals, usually along the heart's axis. They are then subtracted from the EMG signals.

- Cross Talk: This refers to the interference introduced by the muscles that are not being monitored. These interferences can be reduced by intelligently deciding on the size of the electrodes and the distance between their placement. This background EMG activity can be represented mathematically by (1).

$$
T_{b}^{2}=R_{b}^{2}+T_{i}^{2}+O_{b}^{2}
$$

$\mathrm{T}_{\mathrm{b}}$ is the background EMG at the target.

$R_{b}$ is crosstalk from remote muscles.

$\mathrm{T}_{\mathrm{i}}$ is the intrinsic noise from the target.

$\mathrm{O}_{\mathrm{b}}$ is crosstalk from other muscles.

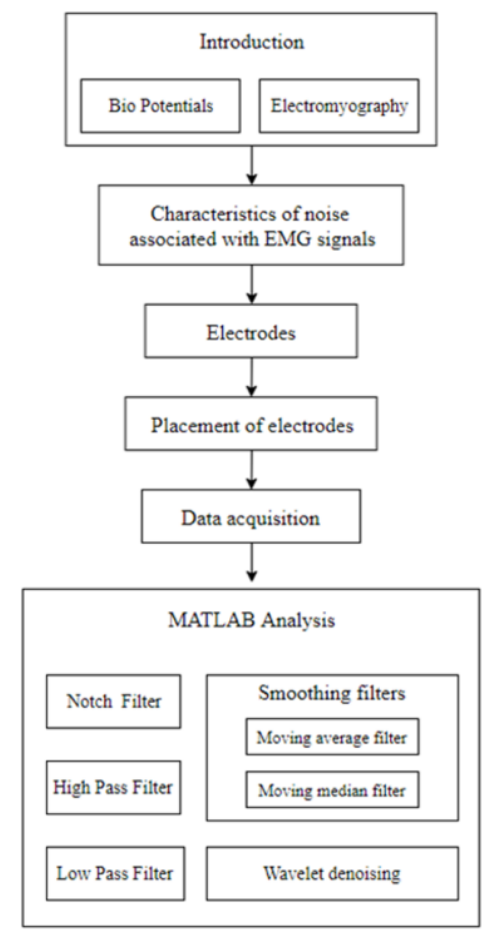

Figure 1: Block Diagram flow

\section{B. Electrodes}

Electrodes are used to convert the ionic potentials into electrical signals that is suitable for processing by electronic circuits. Interactions between the electrons in the electrode and the ions of the body facilitates transduction of the signal [6]. For the purpose of our paper we have used silver/silver chloride electrodes because of its low half-cell potential and low impedance. The characteristics of these electrodes are similar to that of perfectly non-polarizable electrodes. These electrodes have less noise compared to an equivalent polarizable electrode. The electrode has a silver base upon which a coating of silver chloride is applied. This is enclosed by a conductive gel that enhances conductivity and also reduces junction potential [7].
In Figure 2. $R_{d}$ and $C_{d}$ represent the impedance of the electrode-electrolyte interface. $\mathrm{R}_{\mathrm{S}}$ refers to the resistance of the electrolytic gel used and the electrode material itself. $E_{\mathrm{hc}}$ is the half-cell potential of the electrode $(\sim 220 \mathrm{mV})$ [6]. The effective impedance can be represented by (2).

$\frac{R_{d}}{1+j \omega C_{d} R_{d}}+R_{s}$

\section{METHODOLOGY}

\section{A.Placement of electrodes}

Three electrodes are used for EMG measurement. For our purposes we have used the action of palm squeezes for our test. The flexor carpi radialis is a muscle present in the human arm that is most sensitive to this motion. Two of the electrodes are used to directly measure the EMG signals, the other electrode is used as a reference electrode. It is attached to the elbow region of the arm. This is because this region is predominantly bone which tends to act as more electrically neutral than regions of the body with muscular tissue [8]. To reduce the noise introduced by crosstalk, Minimum Crosstalk Area (MCA) is defined. It is the surface that has been defined by Basmajian \& Blumenstein where crosstalk versus co-contraction is minimum [8]

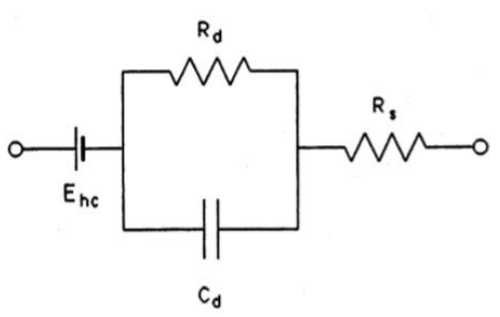

Figure 2: Representation of electrode electrolyte interface of biopotential electrode

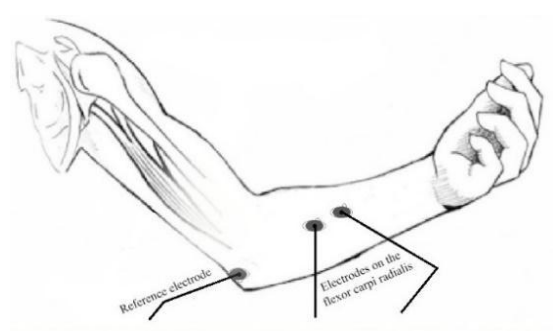

Figure 3: Placement of electrodes on the arm

\section{A.Data acquisition}

The analysis and filtering has been performed on EMG signals extracted using an AD8232 Module connected to the electrodes that were attached to the arm. The data was then collected from the COM port of a computer system using the freeware CoolTerm. This raw data was then converted into a .txt file format. We also performed the same analysis on raw data collected from an online

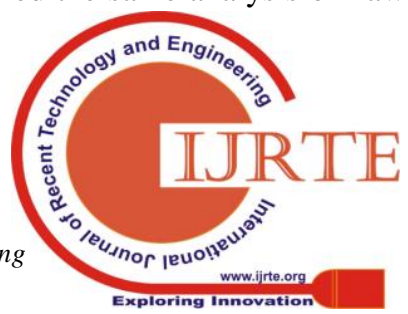


database. These signals were collected using a Medelec Synergy N2 EMG Monitoring system. These were then given as inputs to the MATLAB Program.

\section{B.MATLAB Analysis}

The data is defined by a matrix [Ai1 Bi2]. The column Ail corresponds to the amplitude of the voltage readings from the electrodes and the other column $\mathrm{Bi} 2$ consists of their corresponding time intervals. This data is plotted to obtain the raw EMG signal waveform in time domain. (Figure 4).

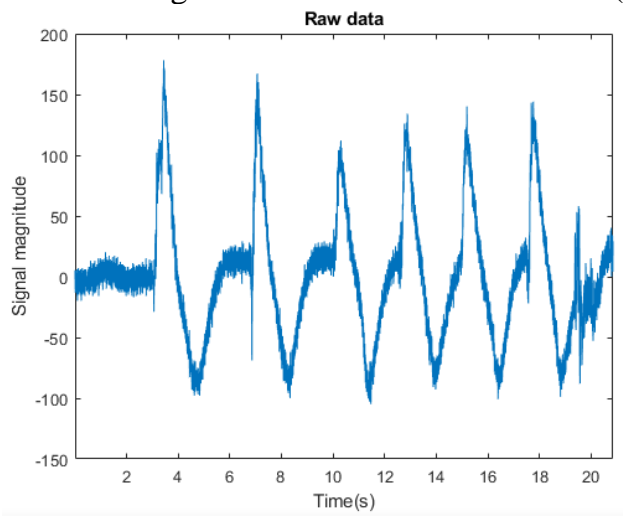

Figure 4: Raw data (Time Domain)

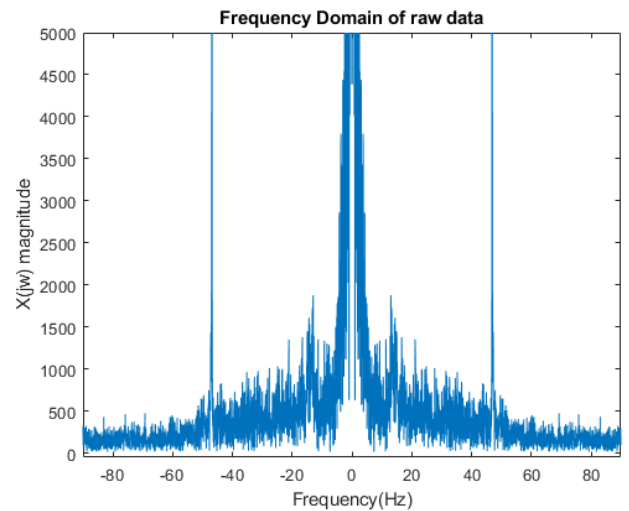

Figure 5: Raw data (Frequency Domain)

The Fast Fourier Transform (FFT) is used to compute the frequency spectrum. The plot of the frequency domain signal is made (Figure 5). Since most of the noise components lie within the frequency and amplitude range of the EMG signals, it is difficult to visualise and identify those components just by inspection. Different filtering techniques are used to achieve this. In general, a discrete time filter's output is given by a rational transfer function defined by numerator and denominator coefficients. Maximum of these coefficients gives the order of the filter. Different filters have been applied to overcome specific noises associated with EMG.

\section{1) Notch Filter}

It can be observed from the frequency distribution of the signal (Figure 5) that there exists interference at approximately the $50 \mathrm{~Hz}$ range of the signal. This is due to external electromagnetic interference more specifically Power Line Interference. Observing the frequency distribution for multiple trials it was concluded that designing a band stop filter at the frequency range of $47 \mathrm{~Hz}$ was more effective than the typical $50 \mathrm{~Hz}$ band stop filters used in popular literature on removing PIL noise.

A band stop or band reject filter passes all frequencies except those that lie between the two cut off frequencies. Since our purpose requires the filtering out a small set of frequencies around $47 \mathrm{~Hz}$, a notch filter is used. A notch filter has a very narrow and highly attenuated stop band. A standard notch filter is given by (3).

$$
H(s)=\frac{s^{2}+\omega_{o}^{2}}{s^{2}+\omega_{c} s+\omega_{o}^{2}}
$$

With the central rejected frequency, $\mathrm{w}_{\mathrm{o}}$ and the width of the rejected band, $\mathrm{w}_{\mathrm{c}}$. IIR Notch filters are designed with the stopband frequencies as $42 \mathrm{~Hz}$ and $52 \mathrm{~Hz}$. The frequency domain signal after notch filtering is represented in (Figure 6). IIR stands for Infinite Impulse Response. Equation of an IIR response is given by (4).

$$
y[n]=\frac{1}{a_{0}}\left(\sum_{i=0}^{M} b_{i} x[n-i]-\sum_{j=1}^{N} a_{j} y[n-j]\right)
$$

Where $\mathrm{M}$ is the feedforward filter order, $\mathrm{N}$ is the feedback filter order, $b_{i}$ are the feedforward coefficients, $a_{j}$ are the feedback coefficients, the input signal is $x[n]$ and the output response, $\mathrm{y}[\mathrm{n}]$.

\section{2) High Pass Filter}

Motion artifacts and random firing of motor neurons due to inherent instability of neurons account to noise which lies below $20 \mathrm{~Hz}$. Use of high pass filters reduce this. High pass filters pass the frequencies above the cut off frequency and attenuate those below (Figure 7). IIR filters are designed with corner frequency as $20 \mathrm{~Hz}$.

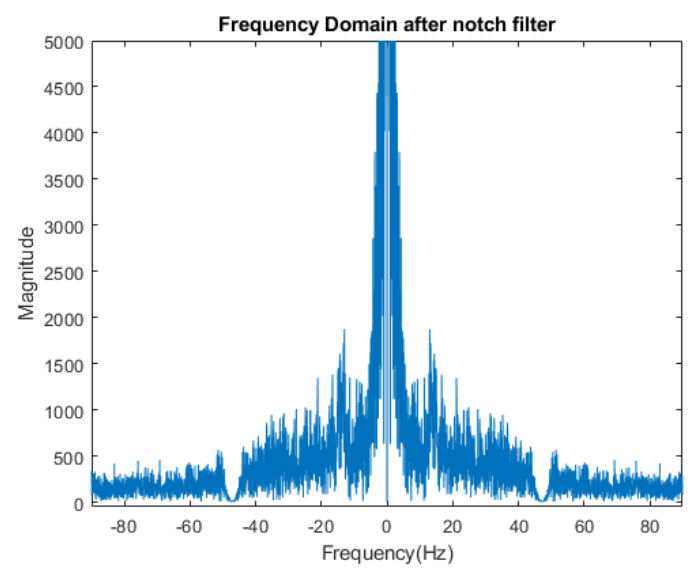

Published By: 
Figure 6: Signal after notch filtering (Frequency domain)



Figure 7: Signal after high pass filtering (Frequency domain)

EMG signals can range from $20 \mathrm{~Hz}$ to $1000 \mathrm{~Hz}$. However, most of the useful components of the signal are concentrated below $200 \mathrm{~Hz}$ and noise occupies the higher frequencies For our study, we implemented a low pass filter with a corner frequency below $100 \mathrm{~Hz}$ to reduce computational complexity. (Figure 8). A band pass filter can also be implemented to achieve the same effect, having a lower passband frequency equal to $10 \mathrm{~Hz}$ and the upper passband frequency equal to the cut-off of the low pass filter.

\section{3) Smoothing Filter}

Sudden variations in the signal due to unwanted movements can also be reduced by applying smoothing techniques. These techniques include moving average filters and moving median filters. The moving average and moving median filters use a window of a defined size and slide this window along the data, computing the average and median of the data contained in each window respectively. In simple moving average filters, all the samples are weighted equally. (Figure 9). Output response of a moving average filter having a window length of ' $\mathrm{W}_{\mathrm{S}}$ ' is given by (5).

$$
y(n)=\frac{1}{W_{s}}\left(x(n)+x(n-1)+. .+x\left(n-\left(W_{s}-1\right)\right)\right)
$$

Window size is chosen to be 4 for both filters. The value of the coefficients is made equal to $1 / \mathrm{W}_{\mathrm{S}}$. On the other hand, weighted average filters follow the binomial expansion of $[1 / 2$ $1 / 2]^{\mathrm{n}}$. The coefficients of this binomial filter convolve $\left[\begin{array}{ll}1 / 2 & 1 / 2\end{array}\right]$ with itself iteratively $n$ times. The value of $n$ is chosen to be 2 , making the window size equal to 4 .

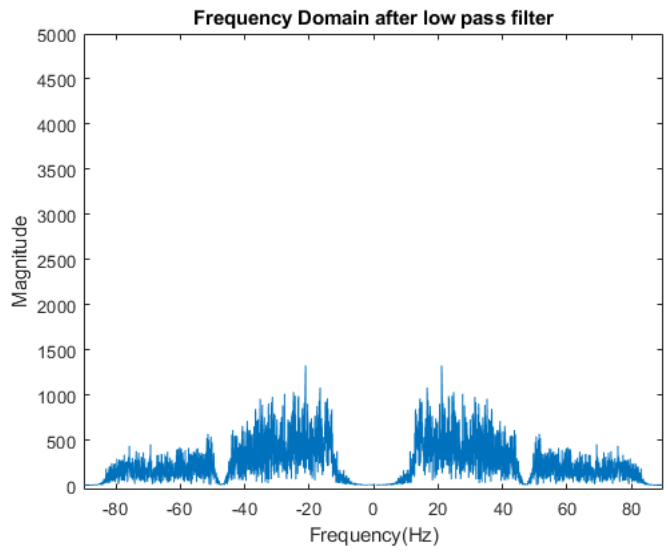

Figure 8: Signal after low pass filtering (Frequency domain)

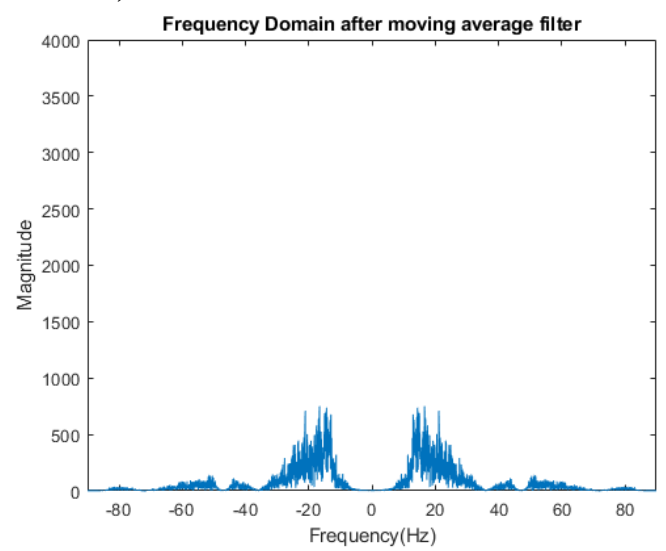

Figure 9: Signal after moving average filter (Frequency domain)

\section{4) Wavelet Filtering}

By using wavelets, the signal can be separated into frequency elements that are time-variant. They localise features in the data into different scales. The useful signal components are concentrated in the large magnitude wavelet coefficients whereas the noise is mainly concentrated in the smaller magnitude wavelet coefficients and can be removed [9].

The denoising procedure involves three steps: decomposition, thresholding and reconstruction. In decomposition, the signal is separated into $\mathrm{M}$ levels. A threshold is selected and applied to the coefficients in each of the above levels. The reconstruction is performed by making use of both the original coefficients of level $\mathrm{M}$ as well as the revised coefficients of 1 to M levels. For our study, a sym4 wavelet of the symlet family (Figure 10) is chosen and the output of denoising is computed using thresholding techniques.

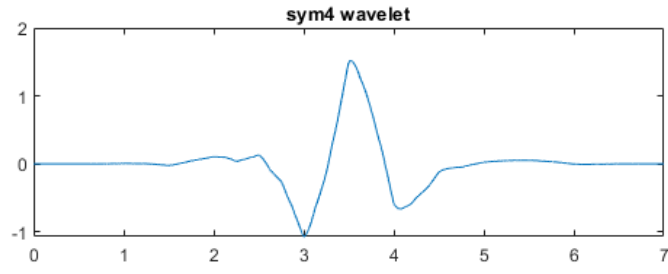

Figure 10: Sym4 wavelet 


\section{RESULTS}

The mean absolute value (MAV) of the signal is found by the method of convolution. K-means clustering is applied to identify different regions using a clustering window of defined size. The clustering is used to partition the data into 2 clusters, one corresponding to the regions of contraction in the muscle and another corresponding to the baseline without movement of muscles. The final output is plotted (Figure.11). In the output waveform, pulses are observed whenever there is a muscular contraction.

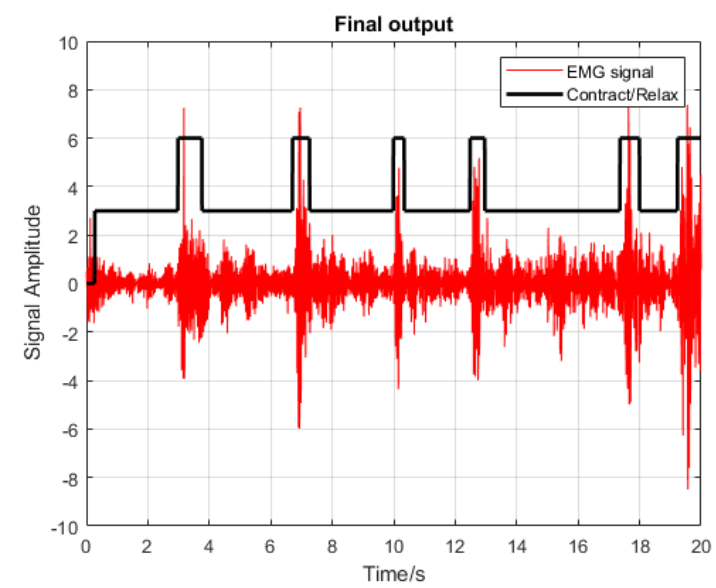

Figure 11: Final plot with pulses representing contraction (Time Domain)

\section{CONCLUSION AND FUTURE SCOPE}

In this paper, we provide a brief description on research done on electromyography signals and work carried out by us to virtually gather useful information from it through MATLAB. Each type of noise is considered separately, and different filtering methods are performed to increase signal to noise ratio. Notch filter, high pass and low pass filter, smoothing methods and wavelet denoising are performed on the raw signal. We are accurately able to classify contractions and relaxations in the muscle.

The limitations to this method are defined by the hardware constraints. Optimised processing techniques can be implemented, but inherent noise in the acquisition circuit cannot be eliminated. In this paper, we have discussed the processing of acquired and stored data. But it can be extended to work in real time conditions. This kind of data can be sent to the cloud and the doctor can access the patient's data online and provide suitable diagnosis

\section{REFERENCES}

[1] J. W. Clark Jr., "The Origin of Biopotentials" in J. G Webster, "Medical Instrumentation", 4th ed, John Wiley and Sons, New York, pp. 126-87, 2010

[2] C. Steele, "Applications of EMG in Clinical and Sports Medicine", InTech, Croatia, December 2011.

[3] C. Heckman and R. M. Enoka, "Physiology of the motor neuron and the motor unit", pp. 119-147, 2004

[4] M.B.I.Reaz, M.S Hussain, F. Mohd-Yasin, "Techniques of EMG signal analysis: Detection, processing, classification and Applications", 2006
[5] H.W.Tam, J.G. Webster, “Minimizing electrode motion artifact by skin abrasion”, IEEE Trans. Biomed. Eng., 1977

[6] M. R. Neuman, "Biopotential Electrodes", in J. G Webster, "Medical Instrumentation": 4th ed, John Wiley and Sons, New York, pp. 189-240, 2010

[7] G. J. Janz, D. J. G. Ives, "Silver, Silver Chloride Electrodes", 1968

[8] Y. Blanc, U. Dimanico, "Electrode Placement in Surface Electromyography (sEMG) "Minimal Crosstalk Area" (MCA)", 2010

[9] X. Zhang, Y. Wang, R.P.S. Han, “Wavelet transform theory and its application in EMG signal processing”, Yantai, China, September 2010. 